\title{
Optimal Fuzzy and Dynamics Design of Ecological Sandwich Panel Vessel Roofs
}

\author{
Heikki Martikka ${ }^{1}$ and Erkki Taitokari ${ }^{2}$ \\ ${ }^{1}$ Himtech Oy, Ollintie 4, 54100 Joutseno, Finland \\ ${ }^{2}$ Oy Scanfibre Ltd., Liisankatu 26, 55100 Imatra, Finland \\ Correspondence should be addressed to Heikki Martikka, heikki.martikka@pp.inet.fi
}

Received 16 December 2010; Accepted 27 July 2011

Academic Editor: Kemal Kilic

Copyright ( $) 2011$ H. Martikka and E. Taitokari. This is an open access article distributed under the Creative Commons Attribution License, which permits unrestricted use, distribution, and reproduction in any medium, provided the original work is properly cited.

In this study the basic engineering principles, goals, and constraints are all combined with fuzzy methodology and applied to optimally design sandwich panel circular plate roofs for large vessels loaded statically and dynamically. These panels are made up of two stiff, strong veneer skins separated by vertical and peripheral stiffener plates. Advantages are high strength, lightweight, and sustainability. In the present approach, first the goals and constraints of the end user are identified and expressed as decision variables which are formulated using the engineering variables for materials, geometry, and function. Then same consistent fuzzy satisfaction functions are formed over the desired ranges to suit the customer's desires. The risk of extreme dynamic loadings exciting resonance is studied by natural frequency and mode analysis by FEM and analytical models. The results show the most critical locations and give guidelines for innovative remedies of the concept before detailed FEM analyses to finalize the design.

\section{Introduction}

Sandwich panels with stiff light cores offer many advantages compared to solid products such as high bending and buckling load-bearing capacities and weight saving without sacrificing reliability. Especially in large industrial roofs, these properties promise benefits provided the structures are optimally designed also.

This case study is based on an industrial design task of a very large circular plate veneer sandwich roof.

The conventional analytical optimisation methods are cumbersome and not suitable for engineering design work as discussed by Gibson and Ashby [1] and Ashby [2] from the point of view of optimal material selection. The present approach is based on the fuzzy goal aggregation ideas in design optimisation presented by Diaz [3]. In this study the same fuzzy formulation is used consistently to describe the goals and constraints of the end user.

In engineering optimisation at concept stage, most tasks are highly nonlinear, and fuzzy and design variables are few and discrete. This approach is used by Martikka and Pöllänen [4], who have applied multiobjective optimisation combined with technical laws and heuristics. Martikka [5] has applied fuzzy optimum methodology to the design of industrial composite structures with the goal of maximising the customer satisfaction. Taitokari and Martikka [6] have studied the dynamics design of vessels of fibre-reinforced plastic with steel shafts for fluid mixing using microstructural design. The design of wood structures is based on the micro- and macrostructural modelling of wood which is discussed in [1] and on the basic theory of analysis and performance of fibre composites by Agarwal and Broutman [7]. The global megatrends of our technological civilisation studied by Marchetti [8] are important guidelines for doing ecodesign engineering work and choosing sustainable materials like wood. Hassan [9] discusses value-focused thinking on the environmental selection of wall structures. Ecological wood surface protection methods developed recently [10] promote optimal utilisation of wood in very large industrial roofs and also large buildings and bridges. In this task basic engineering as presented by Blake [11] is needed. For solving complex structural engineering tasks, the FEM is an indispensable tool [12]. Using it may overcome the know-how barriers of purely 


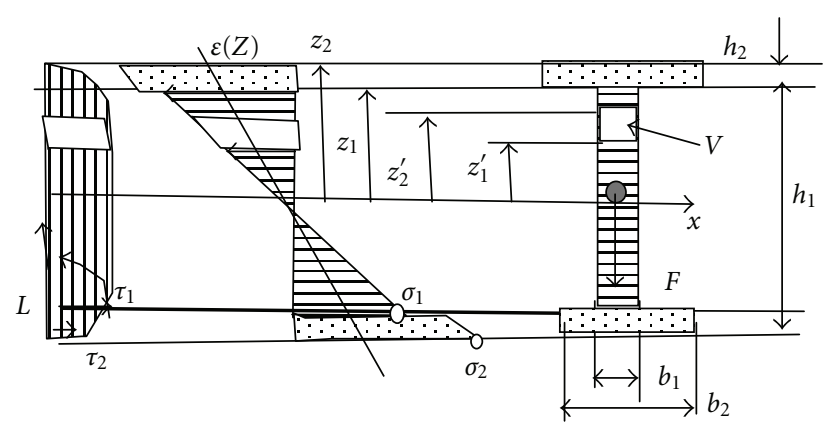

Figure 1: Stress definitions. Material removal from the web can be activated at a defined area $V$.

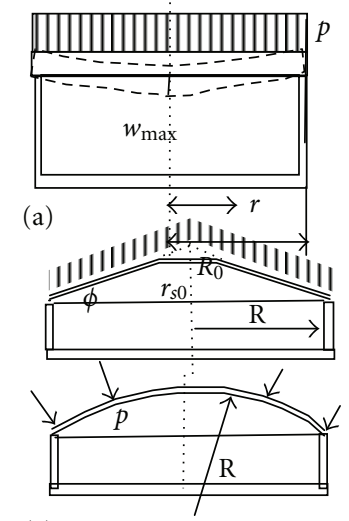

(c)

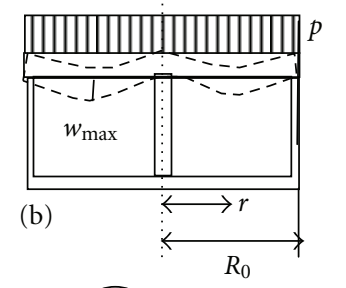

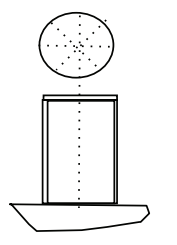

(e)

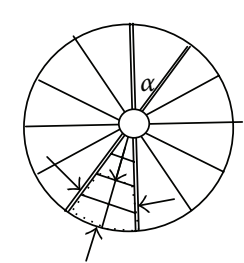

FIgURe 2: Roof models for cylindrical vessels. (a) Model 1 with no centre support. (b) Model 2 with support. (c) Cone and sphere forms. (d) Stresses on face.

analytical structural dynamics theory as discussed by Soedel [13].

One future goal is to utilise biomimicry to copy the ingenious optimal designs in nature into technological products.

\section{Basic Mechanics of Sandwich Panel Roofs}

Basic mechanics is needed to understand and to utilise the sandwich design. Basic concepts for sandwich roofs are illustrated in Figures 1-3.

Large circular roofs are commonly made as flat circular plates, cones, spherical, or some curved form as shown in Figure 2.

Geometry definitions of a roof sector are shown in Figure 3.

\section{Design Variables}

Design variables are classified into three main types.

3.1. Functional Design Variables. Among these are loads, supports, reliability, and factors of safety. Load is external static pressure $p=0.01 \mathrm{MPa}$ on the top of the roof. It can

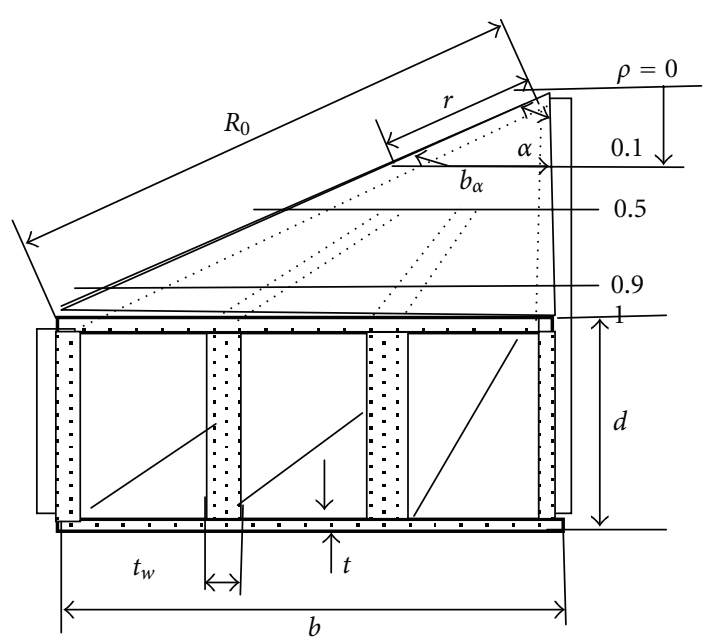

Figure 3: Geometry of a roof sector. Number of stiffeners is $n_{w}=3$.

be changed to design variable. Self-weight pressure due to veneer is about $p_{\text {self }}=3 \cdot \rho_{\text {veneer }} \cdot \operatorname{tg}=0.001 \mathrm{MPa}=0.1 p$. Now it is considered as included in the total load caused by snow loads.

3.2. Geometric Variables. Outer radius of roof is $R_{0}=a=$ $10 \mathrm{~m}$. Radius from centre is $r$, and the relative radius $\rho=$ $r / R_{0}$. Design variable options are shown in Table 1.

3.3. Material Variables. Material variables for wood and for veneer are shown in Table 2 to illustrate its variability of strength properties. Now only veneer is used in the roof as main load-bearing material. Allowable design value strengths are used as parts of the factors of safety of their decision variables.

\section{Stresses and Deflections}

4.1. Roof with No Centre Support. The dominant stress state in the roof sandwich is the face normal stress. Bending stresses at two heights from neutral plane below and on top of the flange are

$$
\sigma_{1}=\frac{M}{D} z_{1} E_{1}, \quad \sigma_{2}=\frac{M}{D} z_{2} E_{2}
$$

Here $M=M_{x} b(\mathrm{Nm} / \mathrm{m})(\mathrm{m})=(\mathrm{Nm})$ is true moment and $M_{x}(\mathrm{Nm} / \mathrm{m})$ is line moment, $b$ is width of the plate, $E_{1}$ and $E_{2}$ are Young's moduli, and $D$ is bending stiffness. For a material layer $k$, one obtains

$$
\sigma_{k}=E_{k} \varepsilon(z)=E_{k}\left(\varepsilon_{0}+\beta z\right)
$$

Thus

$$
\sigma_{k}=\frac{N}{\Sigma E A / E_{k}}+\frac{M z}{\Sigma E I / E_{k}} .
$$


TABLE 1: Design variable options.

\begin{tabular}{lccccccccc}
\hline Variable & 1 & 2 & 3 & 4 & 5 & 6 & 7 & 8 & 9 \\
\hline$d($ id), height $(\mathrm{m})$ & .200 & 0.22 & 0.24 & 0.26 & 0.30 & .34 & 0.36 & 0.40 & 0.45 \\
$t(\mathrm{itt})$, face $(\mathrm{m})$ & 0.01 & 0.015 & 0.02 & .025 & 0.03 & & & & \\
$n w(\mathrm{iw})$, radial webs & 1 & 2 & 3 & 4 & - & & & \\
$\rho($ irho $)=r / R_{0}$ & 0.1 & 0.5 & 0.9 & & - & & & \\
\hline
\end{tabular}

TABle 2: Material selection data for optimisation.

\begin{tabular}{lccccccccc}
\hline E, $\mathrm{MPa}$ & $\mathrm{R}, \mathrm{MPa}$ & $\mathrm{Im}=2, E$ & $\mathrm{Im}=2, R$ & $\mathrm{Im}=5, E$ & $\mathrm{Im}=5, R$ & $\mathrm{Im}=3, E_{\text {wood }}$ & $\mathrm{Im}=3, R_{\text {wood }}$ & Veneer, $E$ & Veneer, $R$, ifa $=1$, face \\
\hline$E_{L}$ & $\sigma_{\mathrm{LU}}$ & 35000 & 800 & 3500 & 80 & 10000 & $90 \mathrm{t}, 43 \mathrm{c}$ & 5000 & 50 \\
$E_{T}$ & $\sigma_{\mathrm{TU}}$ & 8000 & 100 & 3500 & 80 & 500 & 4 & 5000 & 60 \\
$E_{z}$ & $\sigma_{\mathrm{zU}}$ & 800 & $80 \mathrm{c}, 12 \mathrm{t}$ & 3500 & 80 & 800 & 6 & 5000 & 20 \\
$G_{\mathrm{LT}}$ & $\tau_{\mathrm{LTU}}$ & 2500 & 25 & 1300 & 30 & 650 & 7 & 2000 & 20 \\
$G_{\mathrm{Tz}}$ & $\tau_{\mathrm{TzU}}$ & 2500 & 25 & 1300 & 30 & 50 & 7 & 2000 & 20 \\
$G_{\mathrm{zL}}$ & $\tau_{\mathrm{zLU}}$ & 2500 & 25 & 1300 & 30 & 650 & 7 & 2000 & 20 \\
\hline
\end{tabular}

Here $E$ is elastic modulus and $R$ is strength property. Material codes are $\operatorname{Im}=2$ for FRP beam, $\operatorname{Im}=5$ for FRP glue, and $\operatorname{Im}=3$ for wood. Main material axes: $L$ : Longitudinal, $T$ : Transverse, $z$ : normal to plate. $c$ : compression value, $t$ : tensile value.

Here $\varepsilon$ is strain at distance $z$ from neutral plane, $N$ is normal force, and $A$ is cross-sectional area. Bending stress and bending stiffness are

$$
\begin{gathered}
\sigma_{b}=\frac{E_{k} M z}{\Sigma E I}=\frac{E_{k} M z}{D}, \\
D=\Sigma E_{k} I_{k}=D^{\prime} b_{\alpha}, \quad I_{k}=\int z^{2} d A_{k} .
\end{gathered}
$$

Deflection of plate at radius $r$ from centre is

$$
\begin{array}{r}
w(r)=\frac{p r_{0}^{4}}{64 D^{\prime}(1+v)}\left[2(3+v) C_{1}-(1+v) C_{0}\right], \\
C_{0}=1-\rho^{4}, C_{1}=1-\rho^{2}, \rho=\frac{r}{R_{0}} .
\end{array}
$$

The stiffness is due to face sheets and the grid of web plates in radial and peripheral directions. It is assumed that the plate is equally stiffened

$$
\begin{gathered}
D=E I=D^{\prime} b_{\alpha}, \quad b_{\alpha}=r \alpha=\rho R_{0} \alpha, \\
E I=(E I)_{\text {faces }}+(E I)_{\text {webs }} .
\end{gathered}
$$

This may be expanded as

$$
E I=\left(E_{f 1}+E_{f 2}\right) b_{\alpha} t\left(\frac{1}{2} d\right)^{2}+n_{w} \frac{t_{w} d^{3}}{12} .
$$

Line moments and shear are

$$
\begin{gathered}
m_{\mathrm{r}}=-D^{\prime}\left(w_{, r r}+\frac{v}{r} w_{, r}\right)=\frac{p r_{0}^{2}}{16 D^{\prime}}(3+v) C_{1}, \\
m_{\mathrm{t}}=-D^{\prime}\left(\nu w_{, r r}+\frac{1}{r} w_{, r}\right)=\frac{p r_{0}^{2}}{16 D^{\prime}}\left[2(1-v)+(1+3 v) C_{1}\right], \\
q_{\mathrm{r}}=-\frac{p r_{0}}{2} \rho, \quad D^{\prime}=\frac{D}{b_{\alpha}} .
\end{gathered}
$$

Some abbreviations are advantageous in moments

$$
\begin{gathered}
m_{r}=\frac{p r_{0}^{2}}{16 D^{\prime}}(3+v) C_{1}=G R, \\
R=(3+v) C_{1}, \quad G=\frac{p r_{0}^{2}}{64 D^{\prime}}, \\
m_{t}=\frac{p r_{0}^{2}}{16 D^{\prime}} T=G T, \quad T=\left[2(1-v)+(1+3 v) C_{1}\right] .
\end{gathered}
$$

The stresses are

$$
\sigma_{r}=\frac{m_{r}}{t d}=\frac{G R}{t d}, \quad \sigma_{\theta}=\frac{m_{\theta}}{t d}=\frac{G T}{t d} .
$$

Shear force is

$$
q_{r}=-\frac{p r_{0}}{2} \rho \Longrightarrow Q_{r \mathrm{tot}}=q_{\mathrm{r}} b_{\alpha} .
$$

Shear stress is maximal at midweb with stress concentration factor $K_{\tau}$

$$
\begin{aligned}
\tau & =K_{\tau} \frac{Q_{r \text { tot }}}{t_{w} d} \Longrightarrow Q[N]=K_{\tau} q\left[\frac{N}{m}\right] b_{\alpha}[m] \Longrightarrow \\
\tau & =K_{\tau} \frac{q b_{\alpha}}{b_{\alpha} d}=K_{\tau} \frac{q_{r}}{d} \\
\tau & =\frac{q_{r}}{d} K_{\tau}=-\frac{1}{2} p \frac{r_{0}}{d} \rho K_{\tau}, \quad K_{\tau}=1.5 .
\end{aligned}
$$

For orthotropic plates the failure criterion of Tsai Hill is applicable [9]

$$
\left(\frac{\sigma_{L}}{\sigma_{\mathrm{LU}}}\right)^{2}-\left(\frac{\sigma_{L}}{\sigma_{\mathrm{LU}}}\right)\left(\frac{\sigma_{T}}{\sigma_{\mathrm{TU}}}\right)+\left(\frac{\sigma_{T}}{\sigma_{\mathrm{TU}}}\right)^{2}+\left(\frac{\tau_{\mathrm{LT}}}{\tau_{\mathrm{LTU}}}\right)^{2} \leq 1,
$$

Glue joint is used between face sheet and webs and its failure criterion is

$$
\left(\frac{\tau_{\mathrm{LT}}}{\tau_{\text {LTUglue }}}\right)^{2} \leq 1, \quad\left(\frac{(1 / 2) p\left(r_{0} / d\right) K_{\tau}}{\tau_{\text {LTUglue }}}\right)^{2} \leq 1 .
$$




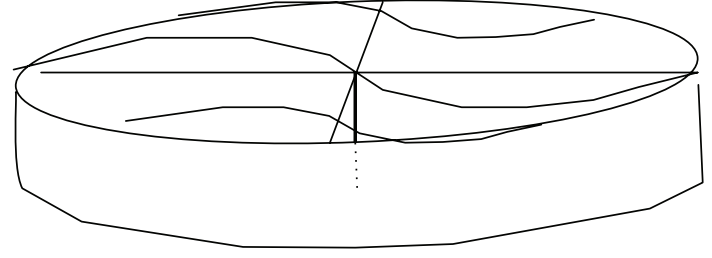

FIGURE 4: Sketch of an assumed lowest mode shape.

4.2. Roof with Centre Support. Now the boundary conditions for the roof are

$$
\begin{gathered}
w(r=0)=0, \quad w\left(r=R_{0}\right)=0, \\
w(r)=G r^{2}\left(\rho^{2}-1\right) .
\end{gathered}
$$

The moments are calculated with (8) and stresses with (10).

\section{Dynamical Modelling of Roof with Centre Support}

5.1. Basic Theory. Calculation of the eigenfrequencies is a difficult mathematical task. Thus some approximate modelling is useful to get fast rough answers. A sketch of a mode shape is illustrated in Figure 4.

According to Soedel [13], the equation of motion of a circular plate can be solved by using the method of separation of variables

$$
w=W \cdot R(r) \Theta(\theta) T(t) .
$$

Here $t$ is time, $\Theta$ is angle, $r$ is radius from centre, and $W$ is the amplitude factor. The radial function is given by the Bessel functions. A trial solution is

$$
\begin{aligned}
& R(r)=A J_{1}(\lambda r)+B Y_{1}(\lambda r) \Longrightarrow \\
& \Theta(\theta)=C_{1} \cos \alpha \theta+C_{2} \sin \alpha \theta, \\
& T(t)=\sin \omega t, \\
& w=W \cdot R \Theta T .
\end{aligned}
$$

The equation for the radial function is

$$
R_{r r}+\frac{1}{r} R_{r}+\left( \pm \lambda^{2}-\frac{\alpha^{2}}{r^{2}}\right) R=0,
$$

where

$$
\lambda^{2}=\omega \sqrt{\frac{\rho h}{D}} .
$$

5.2. Simple Model for Lowest Mode. First a very simple function with two parameters is used to get reasonable estimates

$$
R(r)=r^{p} \sin b r .
$$

One parameter is solved by substituting this to boundary conditions

$$
\begin{aligned}
& w(0)=W \cdot R(0) \Theta T=0, \quad a=R_{0}, \\
& w(a)=W \cdot R(a) \Theta T=0 \Longrightarrow \\
& \sin b a=0, \quad b a=0, \pi, 2 \pi, n \pi .
\end{aligned}
$$

The angle function is made to fit to the assumed shape

$$
\begin{gathered}
\Theta(0)=C_{1} \cos \alpha 0+C_{2} \sin \alpha 0=1 \longrightarrow C_{1}=1, \\
\Theta\left(\frac{1}{2} \pi\right)=C_{1} \cos \alpha \frac{1}{2} \pi+C_{2} \sin \alpha \frac{1}{2} \pi=0, \\
C_{2}=-\frac{\cos \alpha(1 / 2) \pi}{\sin \alpha(1 / 2) \pi}, \quad b=\frac{n \pi}{a} .
\end{gathered}
$$

Thus a simple angle model is

$$
\Theta(\theta)=\cos \alpha \theta-\frac{\sin \alpha \theta}{\tan (\pi / 2) \alpha}, \quad \alpha=\frac{\sqrt{3}}{2} .
$$

The parameters of the simple trial function for describing the first mode can be determined by obtaining first the necessary derivatives

$$
\begin{aligned}
R(r) & =r^{p} \sin b r \\
R_{, r}= & p r^{p-1} \sin b r+r^{p} b \cos b r \\
R_{, r r} & =p(p-1) r^{p-2} \sin b r+2 r^{p-1} p b \cos b r \\
& -r^{p} b^{2} \cos b r .
\end{aligned}
$$

Substitution of these into (18) gives

$$
\begin{aligned}
p(p-1) r^{p-2} \sin b r+2 r^{p-1} p b \cos b r \\
\quad-r^{p} b^{2} \sin b r+p r^{p-2} \sin b r+r^{p-1} b \cos b r \\
\quad+\left( \pm \lambda^{2}-\frac{\alpha^{2}}{r^{2}}\right) r^{p} \sin b r=0 .
\end{aligned}
$$

From this, one obtains

$$
\begin{aligned}
& r^{p-2}\left(p(p-1)-\alpha^{2}\right) \sin b r+r^{p-1}(2 p+1) b \cos b r \\
& -r^{p}\left(b^{2} \pm \lambda^{2}\right) \sin b r=0 \Longrightarrow \\
& p=-\frac{1}{2}, \quad \alpha^{2}=p(p-1)=\frac{3}{4}, \quad b^{2}= \pm \lambda^{2} .
\end{aligned}
$$

The present simple model gives at lowest mode $n=1$

$$
(\lambda a)_{\text {simple }} \approx 3.14 \text {, }
$$

$$
\lambda^{2} a^{2}=\omega a^{2} \sqrt{\frac{\rho h}{D^{\prime}}}= \pm b^{2} a^{2}=n^{2} \pi^{2} .
$$

The bending stiffness per unit length of the plate is

$$
D^{\prime}=\frac{1}{b_{\alpha}} D=\frac{1}{b_{\alpha}} \frac{1}{2} E b_{\alpha} t h^{2}=\frac{1}{2} E t h^{2} .
$$

Density of sandwich plate is

$$
\rho=\frac{m}{V}=\frac{\rho_{w} 2 b_{\alpha} L t}{b_{\alpha} L h}=\frac{\rho_{w} 2 t}{h} .
$$


5.3. More Accurate Model. Now the models by Soedel [13] on the inplane vibrations of circular plates are applied. The solutions are given by the Bessel functions

$$
R=W J_{1}(\lambda r) .
$$

This function satisfies the boundary conditions

$$
\begin{array}{ll}
J_{1}(\lambda a)=0, & \left.\frac{d^{2}}{d r^{2}} J_{1}(\lambda r)\right|_{r=a}=0, \\
J_{1}(\lambda 0)=0, & \left.\frac{d^{2}}{d r^{2}} J_{1}(\lambda r)\right|_{r=0}=0 .
\end{array}
$$

The eigenfrequencies corresponding to the roots of the Bessel function are numbered $m=0,1,2,3$.

The physical meaning is that $m$ represents the number of interior node circles. Now the centre column support constraints to the modes in such a way that the second root probably gives the lowest mode. The lowest roots are $(\lambda a)_{m}=2.403,3.832,5.135,6.379$ [13]. The Eigen frequency corresponding to $m$ is

$$
\omega_{m}=\left(\frac{(\lambda a)_{m}}{a}\right)^{2} \sqrt{\frac{D^{\prime}}{\rho h}} .
$$

Substitution gives

$$
\omega_{m}=\left(\frac{(\lambda a)_{m}}{a}\right)^{2} \sqrt{\frac{(1 / 2) E t h^{2}}{\rho_{w}(2 t / h) h}} .
$$

Thus

$$
\omega_{m}=\left(\frac{(\lambda a)_{m}}{a}\right)^{2} \frac{h}{2} \sqrt{\frac{E}{\rho_{w}}} .
$$

The frequency can be calculated by this using the data for the roof

$$
\begin{gathered}
h=0.3, \quad E=5000 \mathrm{MPa}, \\
\rho_{w}=500 \mathrm{~kg} / \mathrm{m}^{3}, \quad a=10 \mathrm{~m}, \\
(\lambda a)_{2}=3.832, \quad \Longrightarrow f=11 \mathrm{~Hz} .
\end{gathered}
$$

The simple model gave $(\lambda a)_{\text {simple }}=3.14$ and $f_{\text {simple }}=7 \mathrm{~Hz}$. This is less than the theoretical frequency: $f_{\text {theor }}=11$. It is close to the accurate FEM values which are within the range: $f_{\mathrm{FEM}}=10-13 \mathrm{~Hz}$.

\section{Goal and Constraint Formulation}

In the concept stage the essential design variables are few, discrete, and their relationships are highly nonlinear. A fast enough search method is exhaustive learning search. Now all goals and constraints are formulated consistently by one flexible fuzzy function. This is illustrated in Table 3 and Figure 5.

In the design algorithm the satisfaction function is defined for each decision variable $s$ by inputting the left and right limits and two bias parameters $p$. The left skewed
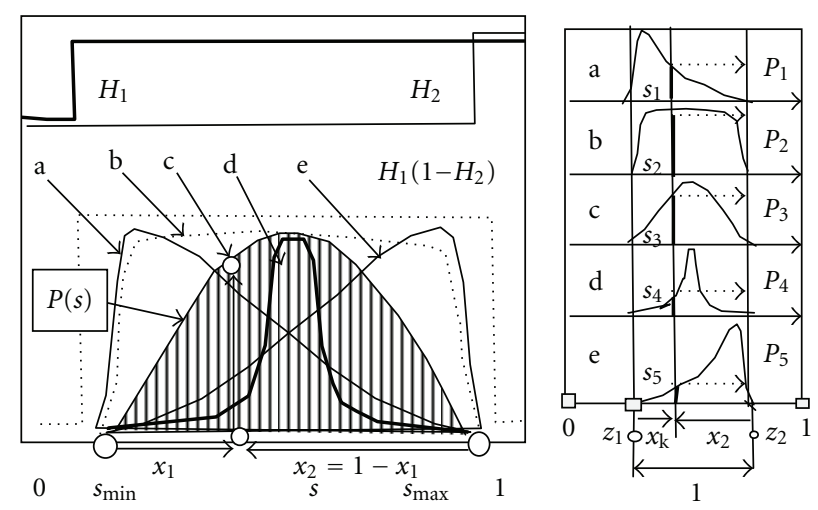

Figure 5: Principle of modelling of the general satisfaction functions.

TABLE 3: Skewness parameter values.

\begin{tabular}{lccccc}
\hline & $\mathrm{a}$ & $\mathrm{b}$ & $\mathrm{c}$ & $\mathrm{d}$ & $\mathrm{e}$ \\
\hline$p_{1}$ & 0.1 & 0.1 & 1 & 5 & 5 \\
$p_{2}$ & 5 & 0.1 & 1 & 5 & 0.1 \\
$x_{1 \max }$ & 0.02 & 0.5 & 0.5 & 0.5 & 0.98 \\
\hline
\end{tabular}

option $a$ is useful to get low-cost designs. Flat shape allows indifferent choice of $s$. The location of maximum can be shifted. The call is CALL $\operatorname{pzz}\left(s_{\min }, s_{\max }, p_{1}, p_{2}, s, P(s)\right)$. The output is the satisfaction function $P(s)$. The total event $s$ is intersection of separate events

$$
s=s_{1} \cap s_{2} \cap s_{3} \cap s_{4} \cap s_{5} \cap s_{6} .
$$

Satisfaction on this event $s$ is

$$
P(s) \Longrightarrow P(s)=P\left(s_{1}\right) \cdot P\left(s_{2}\right) \cdots P\left(s_{n}\right) .
$$

The design goal is to maximise this product.

All design variables are now discrete. A concept is formulated by making trial selections from the lists. Then decision variable and their satisfaction are calculated. Total satisfaction is calculated for each concept alternative. The one with maximal satisfaction is optimal.

\section{Decision Variables}

The desired range for decision variable $s_{k}$ is $R\left(s_{k}\right)=s_{k \min }<$ $s_{k}<s_{k \max }$ and satisfaction on it is $P\left(s_{k}\right)$.

Decision variable $s_{1}$ is face sheet failure risk. Face sheet failure by biaxial stressing is predicted by the Tsai Hill failure criterion in:

$$
F C_{\mathrm{f}}=\left(\frac{\sigma_{L}}{\sigma_{\mathrm{LU}}}\right)^{2}-\left(\frac{\sigma_{L}}{\sigma_{\mathrm{LU}}}\right)\left(\frac{\sigma_{T}}{\sigma_{\mathrm{TU}}}\right)+\left(\frac{\sigma_{T}}{\sigma_{\mathrm{TU}}}\right)^{2}+\left(\frac{\tau_{\mathrm{LT}}}{\tau_{\mathrm{LTU}}}\right)^{2} \leq 1 .
$$

The desired range for the decision variable is from small to 0.7. The bias favours small failure risk

$$
\begin{gathered}
R\left(s_{1}\right)=s_{1, \min }=10^{-5}<s_{1}<s_{1, \max }=0.7, \\
s_{1}=F C_{f}, \quad P_{s}(1)=P\left(s_{1}\right) .
\end{gathered}
$$


Decision variable $s_{2}$ is maximum deflection $w_{\max }$ at midplate. This was obtained for first setting $r=0$ and using the optimal dimensions for $d$ and $t$

$$
\begin{gathered}
\rho=0, \quad C_{0}=1-\rho^{4}=1, \quad C_{1}=1-\rho^{2}=1, \\
w_{\max }=w(\rho=0)=\frac{p r_{0}^{4}}{64 D^{\prime}(1+\nu)}[2(3+\nu)-(1+\nu)] .
\end{gathered}
$$

The desired range for the decision variable is from small to high, 0.7. This high-limit option is used to explore extreme effects due to high loads. But the biased satisfaction function favours desired moderate deflections and excluds unacceptable large values

$$
s_{2}=w_{\max }, \quad P_{s}(2)=P\left(s_{2}\right) .
$$

Decision variable $s_{3}$ is web shear factor of safety. Web shear occurs on $n_{w}$ plates of thickness $t_{w}$ and height $d$ of the total plate. The line shear $q_{r}$ acts on each sector at radius $\rho$. Sector angle is $\alpha=12 \mathrm{deg}$. Shear stress concentration factor is 1.5

$$
\alpha=\frac{2 \pi}{30}, \quad b_{\alpha}=r \alpha=\rho R_{0} \alpha \Longrightarrow Q_{r \text { tot }}=q_{r} b_{\alpha} .
$$

The desired range for the decision variable is from small to 0.2 . The bias favours small value of $s_{3}$

$$
\begin{array}{cl}
s_{3}=\frac{\tau_{\text {web }}}{\tau_{\text {LTuweb }}}, & \tau_{\text {LTuweb }}=1.5 \frac{Q_{r \text { tot }}}{t_{w} d} \frac{1}{n_{w}}, \\
t_{w}=t, & P_{s}(3)=P\left(s_{3}\right) .
\end{array}
$$

Here $\tau_{\text {LTuweb }}$ is the shear strength of the web material, and its thickness is set same as the face sheet thickness $t$.

Decision variable $s_{4}$ is related to the risk of local sheet buckling of the compressed plate in radial direction.

A square plate surrogate model is used to model the buckling risk for the roof plates. A conservative model for the inelastic buckling strength is given by Blak [11]. Now it is assumed that it is freely supported at the stiffened edges. The support factor depends on the edge support stiffness condition. The smallest value is chosen.

$K_{p}=3.62$ for simple-simple edges,

$K_{p}=4.9$ for simple-fixed edges,

$K_{p}=6.3$ for fixed-fixed edges.

The inelastic reduction factor for these edge support types is about $\eta=0.7$. Width is $b$ and thickness is $t$

$$
\begin{gathered}
b=b_{\alpha} \frac{1}{n_{w}}, \quad K_{p}=3.62, \quad \eta=\left(\frac{E_{t}}{E}\right)^{1 / 2} \approx 0.7, \\
S_{\mathrm{cr}}=K_{p} \eta E_{f}\left(\frac{t}{b}\right)^{2} .
\end{gathered}
$$

Elastic modulus definitions are shown in Figure 6.

The desired range for the decision variable is from small to 0.3 . The bias favours small value of $s_{4}$

$$
s_{4}=\frac{\sigma_{r}}{S_{\mathrm{cr}}}, \quad P_{s}(4)=P\left(s_{4}\right) .
$$
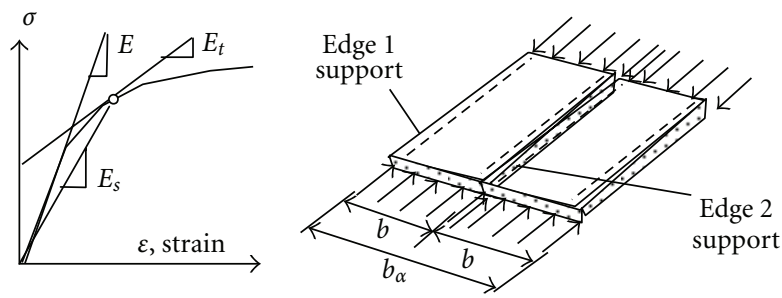

Figure 6: Material model and geometrical definitions.
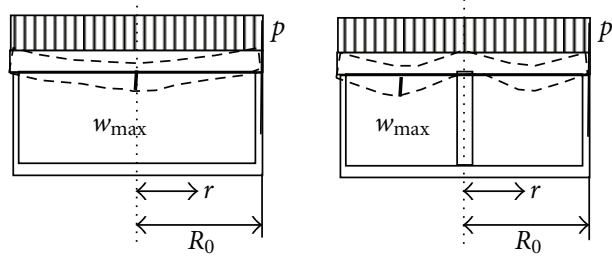

Figure 7: (a) Roof with no centre support, model 1. (b) Roof with centre support, model 2.

Decision variable $s_{5}$ is related to the local sheet buckling of the compressed plate in tangential direction. The desired range for the decision variable is from small to 0.3 . The bias favours small value of $s_{3}$

$$
s_{5}=\frac{\sigma_{t}}{S_{\mathrm{cr}}}, \quad P_{s}(5)=P\left(s_{5}\right)
$$

Decision variable $s_{6}$ is the cost

$$
\text { Cost }=c_{f}\left(m_{f}+m_{w}\right), \quad m_{f}=2 \rho_{f} \frac{1}{2} R_{0}^{2} \alpha t, m_{w}=n_{w} \rho_{f} t R_{0} d .
$$

Satisfaction on it is

$$
P_{s}(6)=P\left(s_{6}\right) .
$$

Decision variable $s_{f}$ is the lowest Eigen frequency

$$
s_{7}=f_{1} .
$$

The desired range is tentatively $f_{1}=5-25 \mathrm{~Hz}$. The frequency for the theoretical model with thickness $h=0.3 \mathrm{~m}$ gave $f_{1}=11 \mathrm{~Hz}$. The statically optimal roof with no centre support had larger thickness $h=0.5$ giving higher frequency $f_{1}^{\prime}=(0,5 / 0.3) \cdot 11=18$. Thus full satisfaction is obtained readily giving

$$
P_{s}(7)=P\left(s_{7}\right) \approx 1 .
$$

\section{Results of Optimisation}

Results are shown in Figure 8 and Tables 4 and 5.

Two different models are considered in Figure 7.

8.1. Model with No Centre Supports. As shown in Figure 8 the satisfaction is maximal at inner radius and decreases towards 


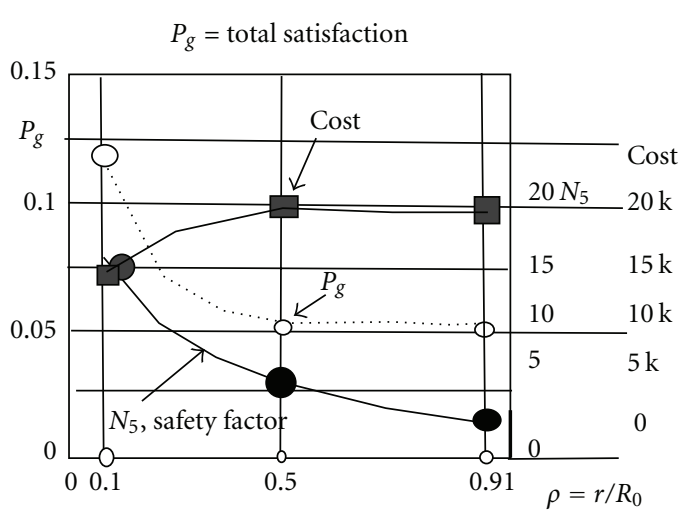

FIgURe 8: Total satisfaction $P_{g}$, cost, and safety factor $N_{5}$ versus relative radius. Model with no roof support.

TABle 4: Results of optimisation. The properties at each relative radius apply to the whole plate. Model 1 with no centre supports.

(a)

\begin{tabular}{lc}
\hline Relative radius $\rho=r / R_{0}$ & $\rho=0.1$ \\
$d(\mathrm{~mm}), t(\mathrm{~mm}), n_{w}$ & $500,30,1$ \\
$w_{\text {max,middle }}(\mathrm{mm}), f_{\mathrm{FEM}}$ & 243 \\
$P_{g}$, total satisfaction & 0.13 \\
$P_{s}(1)$ satisfaction on $s_{1}=\mathrm{FCf}$ & $0.93,0.096$ \\
$P_{s}(2)$ satisfaction on $s_{2}=w_{\max }$ & $0.57,0.243$ \\
$P_{s}(3)$ satisfaction on $s_{3}=\tau_{\text {web }} / \tau_{\text {LTuweb }}$ & $0.76,0.0052$ \\
$P_{s}(4)$ satisfaction on $s_{4}=\sigma_{r} / \mathrm{Scr}, \mathrm{Scr}$ & $.86,0.052,260$ \\
$P_{s}(5)$ satisfaction on $s_{5}=\sigma_{t} / S_{\mathrm{cr}}, N_{5}=1 / s_{5}$ & $0.97,0.072,14$ \\
$P_{s}(6)$ satisfaction on $s_{6}=\operatorname{cost}$ & $0.38,14000$ \\
$\sigma_{\text {Tuf }}, \sigma_{r}, N$ & $50,13.6,3.7$ \\
$\sigma_{\text {Tuf }}, \sigma_{t}, N$ & $60,19,3.15$ \\
$\tau_{\text {web }}, \tau_{\text {LTuweb }}$ & $0.1,20$ \\
\hline
\end{tabular}

(b)

\begin{tabular}{lcc}
\hline$\rho=r / R_{0}$ & $\rho=0.5$ & $\rho=0.9$ \\
$d(\mathrm{~mm}), t(\mathrm{~mm}), n_{w}$ & $500,30,3$ & $500,30,3$ \\
$w_{\text {max }, \text { middle }}(\mathrm{mm}), f_{\mathrm{FEM}}$ & 274 & 300 \\
$P_{g}$, satisfaction & 0.053 & 0.056 \\
$P_{s}(1), s_{1}=\mathrm{FCf}$ & $0.927,0.06$ & $.97,0.063$ \\
$P_{s}(2), s_{2}=w_{\max }$ & $0.5,0.274$ & $0.45,0.3$ \\
$P_{s}(3), s_{3}$ & $0.93,0.044$ & $1,0.14$ \\
$P_{s}(4), s_{4}$ & $0.54,0.11$ & $0.65,0.09$ \\
$P_{s}(5), s_{5}, N_{5}=1 / s_{5}$ & $0.99,0.18,5.5$ & $.91,0.43,2.3$ \\
$P_{s}(6), s_{6}=$ cost & $0.21,19410$ & $0.21,19410$ \\
$\sigma_{\text {Luf }}, \sigma_{r}, N$ & $50,10,5$ & $50,2.6,19$ \\
$\sigma_{\text {Tuf }}, \sigma_{t}, N$ & $60,17,3.5$ & $60,12,5$ \\
$\tau_{\text {web }}, \tau_{\text {LTuweb }}$ & $1.04,20$ & $2.8,20$ \\
\hline
\end{tabular}

outer radius. Main reason is that density of web stiffening is larger at inner radii. This shows that more stiffeners at the outer radius would increase the total satisfaction on the roof.

The cost is now defined as the cost of one sector with angle $\alpha=12 \mathrm{deg}$. The face thickness is variable. The stresses
TABle 5: Results of optimisation. The properties at each relative radius apply to the whole plate. Model 2 with centre support.

\begin{tabular}{lc}
\hline Relative radius $\rho=r / R_{0}$ & $\rho=R_{0} / 1.4=r_{\text {crit }}$ \\
$d(\mathrm{~mm}), t(\mathrm{~mm}), n_{w}$ & $500,10,3$ \\
$w_{\text {max,middle }}(\mathrm{mm}), f_{\mathrm{FEM}}$ & 30 \\
$P_{g}$, total satisfaction & 0.49 \\
$P_{s}(1)$ satisfaction on $s_{1}=\mathrm{FCf}$ & $0.985,0.054$ \\
$P_{s}(2)$ satisfaction on $s_{2}=w_{\max }$ & $0.9982,0.03$ \\
$P_{s}(3)$ satisfaction on $s_{3}=\tau_{\mathrm{web}} / \tau_{\mathrm{LTuweb}}$ & $0.757,0.0052$ \\
$P_{s}(4)$ satisfaction on $s_{4}=\sigma_{r} / S_{\mathrm{cr}}, S_{\mathrm{cr}}$ & $0.838 .0 .05,260$ \\
$P_{s}(5)$ satisfaction on $s_{5}=\sigma_{t} / S_{\mathrm{cr}}, N_{5}=1 / s_{5}$ & $0.85,0.015,65$ \\
$P_{s}(6)$ satisfaction on $s_{6}={ }_{\mathrm{cost}}$ & $0.75,6470$ \\
$\sigma_{\mathrm{Luf}}, \sigma_{r}, N$ & $50,13,3.12$ \\
$\sigma_{\text {Tuf }}, \sigma_{t}, N$ & $60,4,2.7$ \\
$\tau_{\mathrm{web}}, \tau_{\text {LTuweb }}$ & $0.1,20$ \\
\hline
\end{tabular}

are obtained at each radius depending on the geometrical selections. The middeflection is constrained to a desired range. The face and web wall were $0.03 \mathrm{~m}$ and the roof height was $0.5 \mathrm{~m}$. It is assumed that cost is obtained with uniform thickness. From relative radius 0.5 to 1 , the cost, satisfaction, and factor of safety are reasonably constant.

8.2. Model with a Centre Support. Results are shown in Table 5.

8.3. Comparison of Models. Model 1 with no centre support required roof total height $d=0.5 \mathrm{~m}$ and face $t=0.030 \mathrm{~m}$ resulting in maximum deflection of $0.3 \mathrm{~m}$. The cost was $19 \mathrm{k}$ units and satisfaction $P_{g}=0.057$. This deflection was unacceptably large. Therefore this concept was rejected, and only the roof with centre support is considered further in detail.

Model 2 with centre support required $d=0.5$ and the face only $t=0.010 \mathrm{~m}$. Maximum deflection was zero at centre and about $0.007 \mathrm{~m}$ at half radius. This deflection divided by radius is $0.007 / 10=0.0007$ and is acceptable. The satisfaction is $P_{g}=0.49$ and cost $6.5 \mathrm{k}$ units. This shows that adding a center support is profitable if there are no harmful side effects.

In the final design still some detailed changes were made and also a change to slightly conical form.

\section{Results of FEM Checking}

9.1. Results of Static Calculations. Results are shown in Figures 9, 10, and 11. Boundary conditions are shown in Figure 9. The degrees of freedom are maximally six $\left(u_{1}, u_{2}, u_{3}, \theta_{1}, \theta_{2}, \theta_{3}\right)$ along the axes shown. Here the notation of fixity is 1: fixed, 0: free; A: (010000), B: (111000), C: (010101). The roof is freely supported without bending moments at circular opening, the centre with radius $R_{0}=$ $0.5 \mathrm{~m}$, and at the outer radius at the vessel cylinder. The radial sides are given symmetry plane boundary conditions. Static pressure was $p=0.01 \mathrm{MPa}$ at upper plate. Total height 


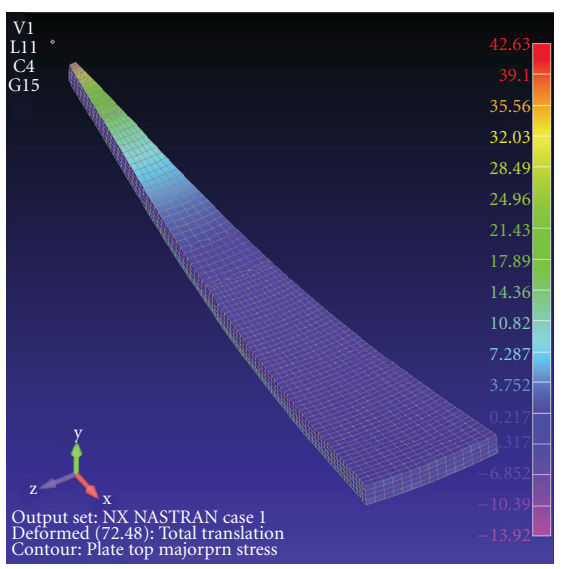

(a)

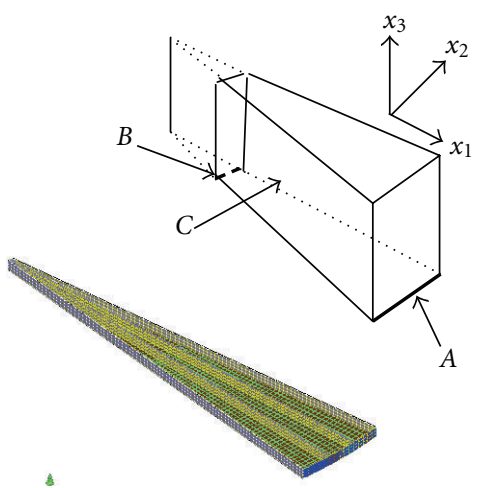

(b)

Figure 9: (a) Deformed geometry and maximal principal stress range +7.5--13.9 MPa. Minimal principal stress range 3.9--33.4. Total deformation is at about $R_{0} / 1.4$ radius and is $72.4 \mathrm{~mm}$ with pressure $p=0.01 \mathrm{MPa}$. (b) Internal web stiffeners and FEM boundary conditions.

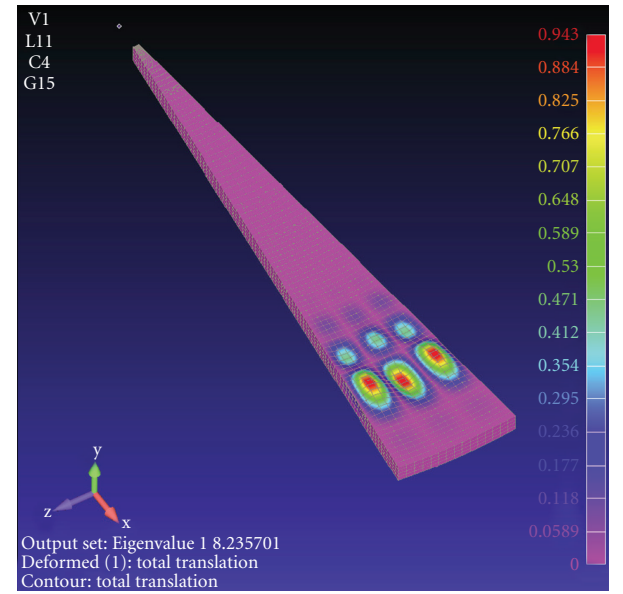

FIGURE 10: The first buckling mode with factor of safety 8.24 . The plates with largest unsupported areas with high compressive stresses buckle first.

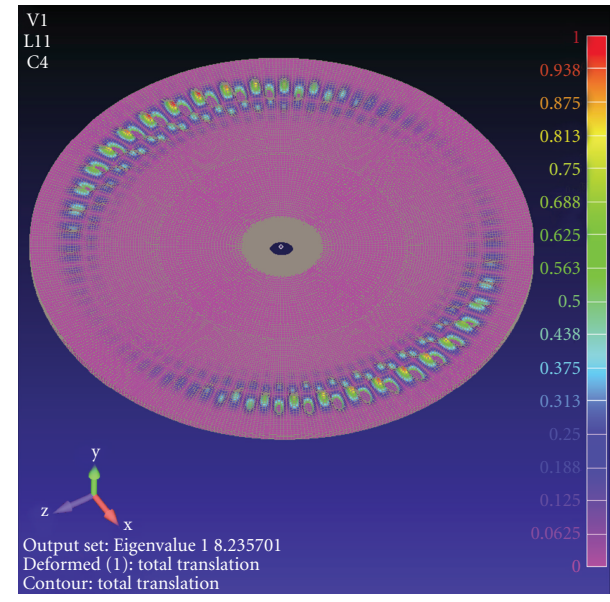

FIGURE 11: The first buckling mode for the whole plate model with factor of safety 8.24 .

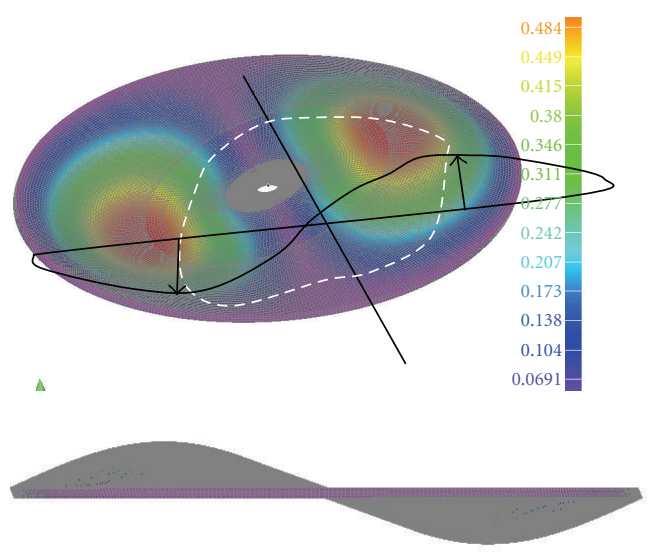

FIGURE 12: The lowest eigen mode corresponding to eigenfrequency of $9.74 \mathrm{~Hz}$. Analytical simple model gave roughly $f=11 \mathrm{~Hz}$.

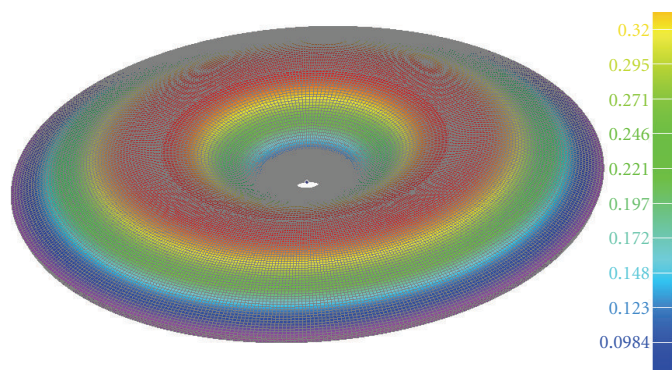

FIGURE 13: The second eigen mode corresponding to eigenfrequency of $10.48 \mathrm{~Hz}$. More complex models are shown in Figures 14 and 15.

$d=0.33 \mathrm{~m}, t=0.03 \mathrm{~m}$ for face and webs, elastic modulus for veneer $E=5000 \mathrm{MPa}$ (Table 2 ), and Poisson's ratio $v=0.3$. The lowest Eigenfrequencies were 10 by FEM and about 11 analytically. They are satisfactorily close to each other considering the large difference between the models. 

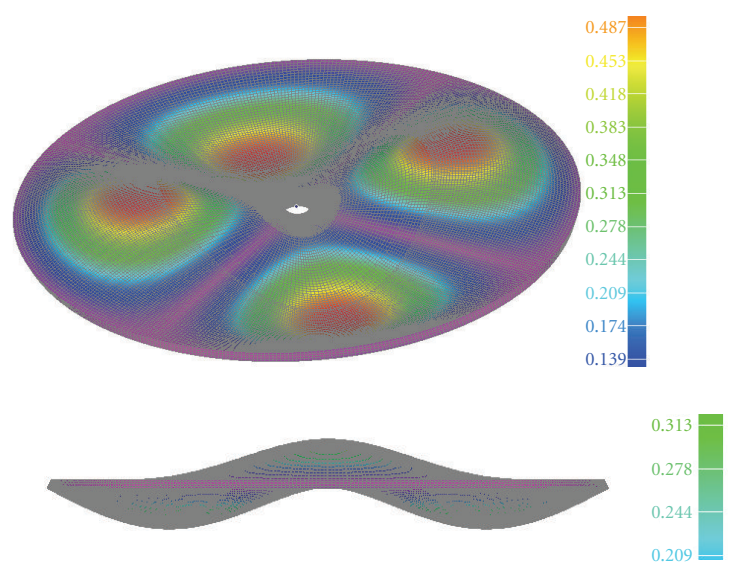

Figure 14: The second eigen mode corresponding to eigenfrequency of $13.01 \mathrm{~Hz}$.

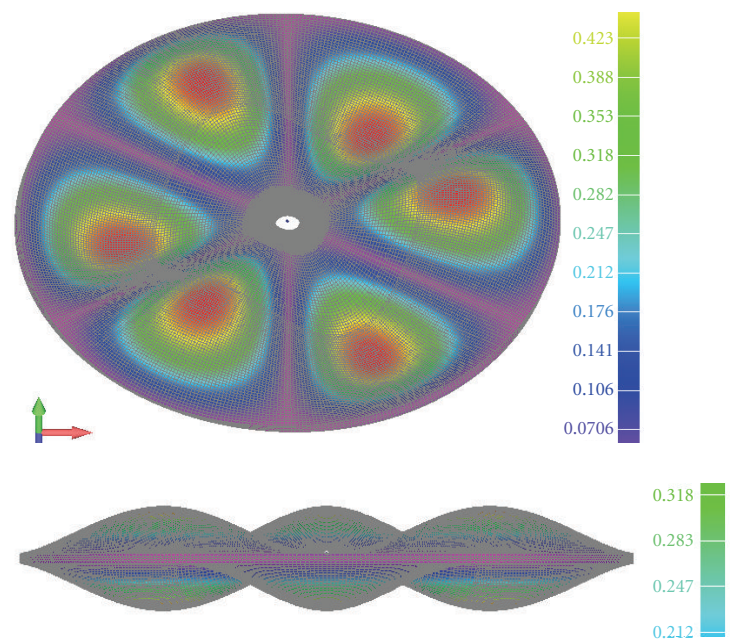

FIGURE 15: The third eigen mode corresponding to eigenfrequency of $16.89 \mathrm{~Hz}$.

9.2. Results of Dynamical Calculations. These are shown in Figures 12, 13, 14, and 15.

\section{Conclusion}

We have combined in this approach basic mechanics with fuzzy goal formulations and heuristics to obtain optimised sandwich panel roof of veneer. In nature, analogous ingeniously designed and biomanufactured structures are already widely and successfully used by animals and plants.

One evident advantage of the present method over the conventional ones is that more useful and reasonable optima can be found since all relevant desires like societal and ecological ones are considered simultaneously during the design. At this stage most of the total life cycle costs and sustainability are determined.

Dynamic methods are important tools to design structures with desired static and dynamic stability under extreme wind or seismic loads. The lowest frequencies by FEM were with rounding 10,11, and $13 \mathrm{~Hz}$. Analytical method gave the lowest as $11 \mathrm{~Hz}$. The utility of the simple models is that they can be used in exploratory optimum concept design. The results show that a reasonable trade-off type optimum between two different constructions is obtained.

The future aim is to generalise this approach of combining basic mechanics with new goal formulations and FEM fine-tuning.

Using this design methodology designers of machines can better mimic the ingenious sustainable solutions found in nature and select the right future megatrends of technology in their design and development work.

\section{Acknowledgments}

The authors are grateful for the support to this research given by the companies Himtech Oy Engineering and Oy Scan Fibre Ltd.

\section{References}

[1] L.J. Gibson and M. F. Ashby, Cellular Solids, Cambridge University Press, New York, NY, USA, 1997.

[2] M. F. Ashby, Materials Selection in Mechanical Design, Elsevier, New York, NY, USA, 3rd edition, 2005.

[3] A. Diaz, "Goal aggregation in design optimization," Engineering Optimization, vol. 13, pp. 257-273, 1988.

[4] H. Martikka and I. Pöllänen, "Multi-objective optimisation by technical laws and heuristics," Memetic Computing, vol. 1, no. 1, pp. 1-2, 2010.

[5] H. Martikka, "Testing and simulation of industrial composite structures for optimizing customer satisfaction," Science and Engineering of Composite Materials, vol. 7, no. 4, pp. 279-286, 1998.

[6] E. Taitokari and H. Martikka, "Dynamics design of vessels of fibre reinforced plastic with steel shafts for fluid mixing," Machine Design. The Monograph of Faculty of Technical Sciences, University of Novi Sad, Chairman Sinisa Kuzmanovic, ADEKO, pp. 21-26, 2009.

[7] B. Agarwal and L. J. Broutman, Analysis and Performance of Fiber Composites, John Wiley \& Sons, New York, NY, USA, 1990.

[8] C. Marchetti, "Swings, cycles and the global economy," New Scientist, vol. 2, 1985.

[9] O. A. B. Hassan, "Application of value-focused thinking on the environmental selection of wall structures," Journal of Environmental Management, vol. 70, no. 2, pp. 181-187, 2004.

[10] http://www.accoya.com/.

[11] A. Blake, Practical Stress Analysis in Engineering Design, Marcel Dekker, New York, NY, USA, 1990.

[12] NX Nastran FEM program.

[13] W. Soedel, Vibrations of Shells and Plates, Marcel Dekker, New York, NY, USA, 2004. 

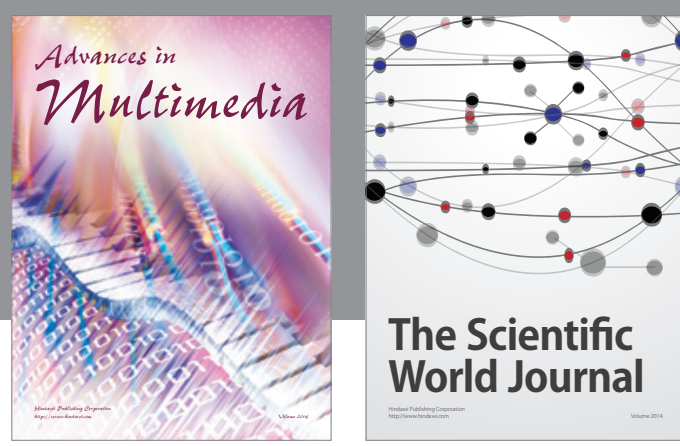

The Scientific World Journal
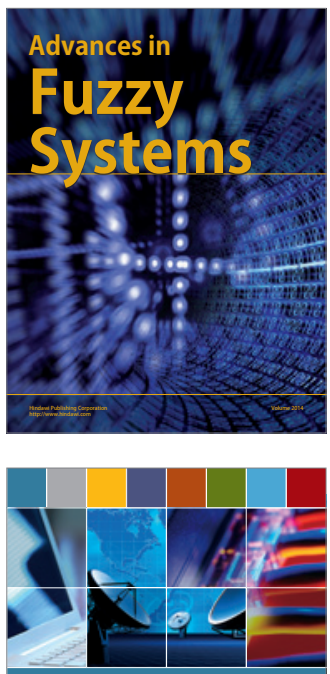

Computer Networks and Communications
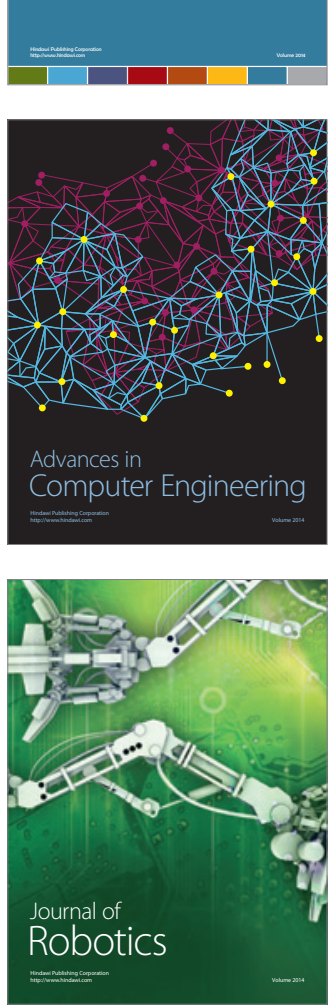
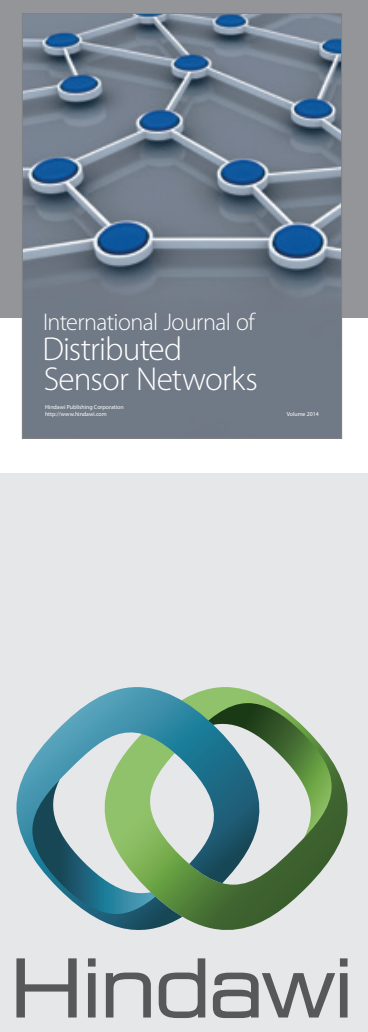

Submit your manuscripts at

http://www.hindawi.com
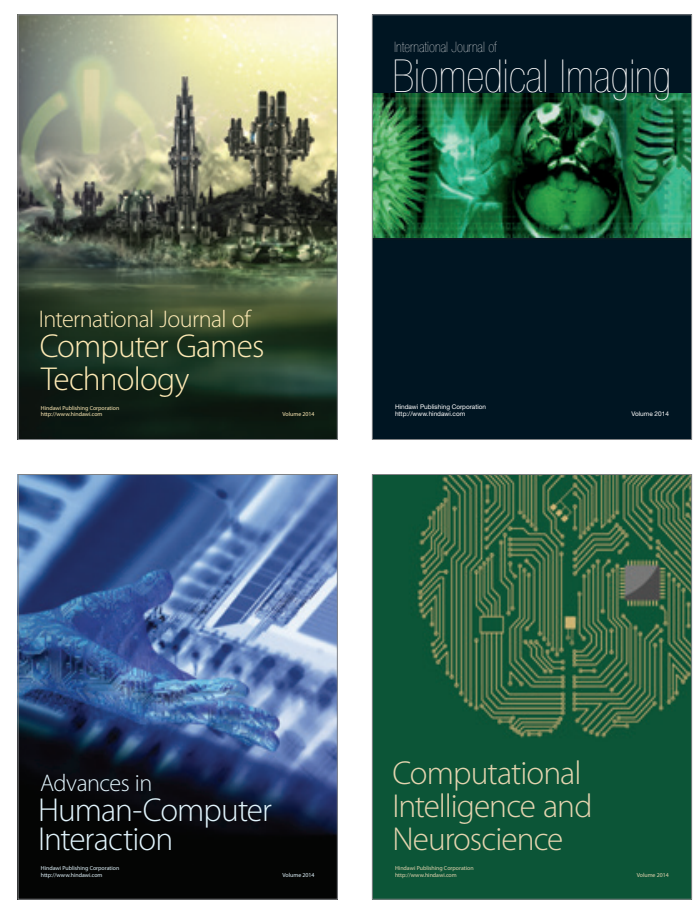
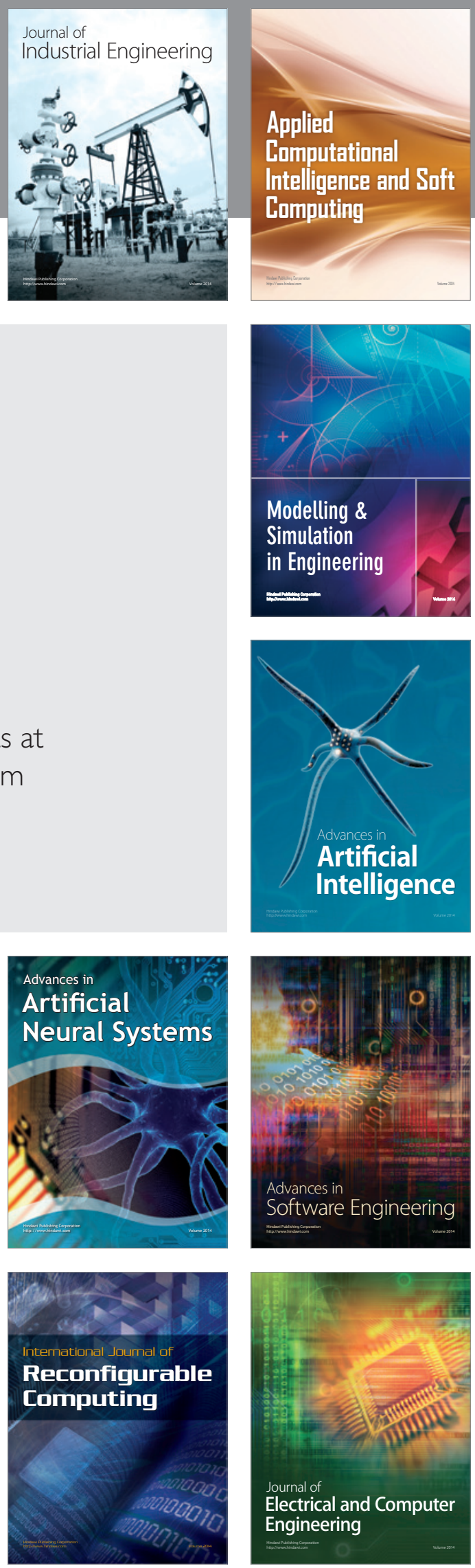